\title{
АЛГЕБРАИЧЕСКАЯ МОДЕЛЬ РАСПРЕДЕЛЕННОЙ ПРОДУКЦИОННОЙ СИСТЕМЫ С НЕЧЕТКИМИ ПРАВИЛАМИ
}

\author{
С. Д. Махортов, М. В. Лещинская \\ Воронежский государственный университет
}

Поступила в редакцию 09.09.2019 г.

\begin{abstract}
Аннотация. Алгебраическая теория LP-структур предназначена для моделирования и оптимизации продукционных и подобных им систем в информатике. Одна из областей ее применения - интеллектуальные системы, основанные на правилах в форме продукций. В предыдущих исследованиях авторами получены результаты, позволяющие обосновывать и автоматизировать решение ряда задач для продукционных систем: эквивалентные преобразования, устранение избыточности, верификация, ускорение обратного вывода.

B настоящей работе вводится и исследуется LP-структура, семантика которой охватывает распределенные нечеткие продукционные системы. Введена терминология FDLP-структур с нечетким бинарным отношением (Fuzzy Distributed LP-структуры). Дано определение логического замыкания нечеткого бинарного отношения, представлена теорема о его существовании. Она позволяет ввести понятие эквивалентных FDLP-структур, соответственно в приложениях - эквивалентных баз знаний. Сформулирована теорема об эквивалентных преобразованиях FDLP-структуры. Ее прикладное значение - способ и его обоснование для автоматизированных преобразований распределенных нечетких баз знаний. Представлено также утверждение о приведении FDLP-структуры к каноническому виду. В приложениях такой формат соответствует множеству хорновских правил.

B рамках рассмотренной расширенной модели возможности теории LP-структур оказываются доступными при построении и исследовании распределенных интеллектуальных систем с нечеткими правилами.

Ключевые слова: интеллектуальная система, нечеткие продукции, LP-структура, логическое замыкание, эквивалентные преобразования, логическая редукция, распределенная система.
\end{abstract}

\section{ВВЕДЕНИЕ}

Информационные технологии играют важную роль в современном мире. Компьютерные системы становятся большими, распределенными, критически значимыми. В результате возрастают значение и актуальность теоретического обоснования корректности и надежности процессов обработки информации в различных прикладных областях. Основу формального построения и исследования моделей компьютерных систем, основанных на различных парадигмах, предоставляют алгебраические структуры [1]. Это в полной мере относится к инженерии знаний, включая широко распространенные в информатике продукционные системы [2-4].

(C) Махортов С. Д., Лещинская М. В., 2019
В последнее десятилетие авторами получены результаты, связанные с логическими системами продукционного типа. Разработана основанная на алгебраических решетках теория LP-структур (lattice production structures) [5], которая обосновывает и эффективно решает задачи эквивалентных преобразований, верификации и минимизации баз знаний. Введен и исследован метод релевантного обратного вывода (LP-вывод) [6], существенно снижающий число обращений к внешним источникам информации. Впоследствии эта теория была расширена для моделирования распределенных продукционных систем [7].

Важную особенность современных интеллектуальных систем представляет нечеткий характер знаний и рассуждений [8]. В связи с данным обстоятельством становится актуальным распространение теории LP-струк- 


\section{С. Д. Махортов, М. В. Лещциская}

тур на нечеткие продукционные системы. Первые шаги в этом направлении были сделаны в работах [9-10]. Введены понятия, характеризующие нечеткость LP-структуры, и изучены некоторые полезные свойства нечеткого LP-вывода. Однако не были рассмотрены такие важные аспекты теории как замыкание, эквивалентные преобразования и эквивалентная минимизация нечетких LP-структур.

Настоящая работа посвящена развитию теории LP-структур для управления распределенными нечеткими базами знаний и моделирования распределенного нечеткого логического вывода.

Введена терминология FLP-структур с нечетким логическим отношением (Fuzzy LP-структуры). Дано определение логического замыкания нечеткого бинарного отношения на решетке, представлена теорема о его существовании. Она позволяет ввести понятие эквивалентных FLP-структур, соответственно в приложениях - эквивалентных баз знаний. Сформулирована теорема об эквивалентных преобразованиях FLP-структуры. Ее прикладное значение - способ и его обоснование для автоматизированных преобразований нечетких баз знаний. Представлено также утверждение о приведении нечеткой LP-структуры к каноническому виду. В приложениях такой формат соответствует множеству хорновских нечетких правил. Введено понятие логической редукции FLP-структуры. Представлены теоремы о ее существовании и способе построения. Для прикладных систем они обосновывают формальную эквивалентную минимизацию нечетких баз знаний.

Построенная модель в заключительном разделе обобщается для моделирования распределенных интеллектуальных систем. Определяется понятие FDLP-структуры (Fuzzy Distributed LP-структура) и изложенные paнее результаты распространяются на распределенный случай.

\section{1. ОСНОВЫ НЕЧЕТКИХ LР-СТРУКТУР}

Моделирование нечетких знаний и рассуждений существенно приближает теорию интеллектуальных систем к решению практи- ческих задач. Во многих современных информационных системах получение «четкого» (точного) решения невозможно, вообще или за приемлемое время. В связи с данным обстоятельством назрела актуальность распространения возможностей теории LP-структур на нечеткие продукционные системы.

Руководствуясь указанной целью, начнем с введения базовых понятий теории нечетких LP-структур. Исходные для настоящего раздела положения теории решеток, нечетких множеств и бинарных отношений изложены, например, в [11-12].

Нечеткое множество $A=\left(F, \mu_{F}\right)$ определяется функцией принадлежности $\mu_{F}: F \rightarrow[0,1]$ на некотором (обычном) множестве $F$. При этом значение $\mu_{F}(a)$ называется степенью принадлежности $a$ к $S$. Соответственно нечеткое бинарное отношение $R$ на множестве $F$ - это нечеткое множество упорядоченных пар элементов из $F$ с заданной функцией принадлежности $\mu_{R}: F \times F \rightarrow[0,1]$.

Нечеткое бинарное отношение $R$ на произвольном множестве $F$ называется реблексивным, если для любого $a \in F$ справедливо $\mu_{R}(a, a)=1$.

В целях моделирования нечеткого логического вывода будем использовать композицию нечетких отношений в классической семантике, а именно - (max-min)-композицию. Для отношения $R$ на множестве $F$ композиция $R^{2}=R \circ R$ определяется следующим образом:

$$
\mu_{R^{2}}(a, c)=\max _{b}\left(\min \left(\mu_{R}(a, b), \mu_{R}(b, c)\right)\right),
$$

где $a, b, c \in F$.

Нечеткое бинарное отношение $R$ на множестве $F$ называется транзитивным, если для любых $a, b, c \in F$ справедливо $\mu_{R}(a, c) \geq$ $\geq \min \left(\mu_{R}(a, b), \mu_{R}(b, c)\right)$. Нетрудно заметить, что свойство транзитивности для отношения $R$ эквивалентно вложению $R^{2} \subseteq R$ в смысле нечетких множеств. Существует также замыкание произвольного нечеткого отношения относительно свойств рефлексивности и транзитивности. Обзор алгоритмов его построения представлен в [13]. 
Известна также задача нахождения транзитивной редукции: для отношения $R$ строится минимальное нечеткое отношение $R^{\prime}$ такое, что его транзитивное замыкание совпадает с транзитивным замыканием $R$. Решению этой задачи посвящена, в частности, работа [14].

Пусть дана атомно-порожденная решетка $\mathbb{F}$, представляющая собой множество всех конечных подмножеств некоторого универсума $F . \mathrm{Ha} \mathbb{F}$ рассматривается (дополнительное) нечеткое бинарное отношение $R$, содержащее $\supseteq$, а также обладающее транзитивностью и дистрибутивностью. Последнее свойство требует уточнения.

Определение 1.1. Нечеткое бинарное отношение $R=\left(\mathbb{F}, \mu_{R}\right)$ называется дистрибутивным, если для любых $A, B_{1}, B_{2} \in \mathbb{F}$ справедливо

$$
\mu_{R}\left(A, B_{1} \cup B_{2}\right) \geq \min \left(\mu_{R}\left(A, B_{1}\right), \mu_{R}\left(A, B_{2}\right)\right) .
$$

Отношение с указанными выше тремя свойствами будем называть продукционно-логическим, или, для краткости - просто логическим.

Определение 1.2. Под нечеткой LP-структурой (FLP-структурой) подразумевается алгебраическая система, представляющая собой решетку $\mathbb{F}$, на которой задано продукционно-логическое отношение $R$.

\section{2. МОДЕЛИРУЕМАЯ ПРЕДМЕТНАЯ ОБЛАСТЬ}

FLP-структура представляет алгебраическую модель нечеткой базы знаний. Поэтому напомним терминологию, связанную с простым видом моделируемых логических систем продукционного типа - экспертными продукционными системами.

Они манипулируют множествами фактов и правил (продукций). Факт представляет некоторое суждение о внешнем мире. Он задается триплетом вида «объект.атрибут = $<$ <зачение>» (например, «термометр.температура = высокая»). В книге [15] для практической реализации продукционных систем триплет сводится к паре «параметр = значение», то есть объект и атрибут агрегируются в единый параметр. В частности, «термометр. температура» и «термометр.изготовитель» считаются разными параметрами. В терминах настоящей работы в факт интегрируются как параметр, та и его значение, при этом факт считается независимым элементом общего множества. В дальнейшем можно реализовать и более сложную конструкцию фактов, расширив описание FLP-структуры.

Продукционная система содержит рабочую память. Это некоторое подмножество фактов, которые на текущий момент считаются выполненными (с коэффициентом уверенности в пределах $[0,1])$. Такое множество называется также базой данных продукционной системы.

Нечеткое правило (продукиия) состоит из предпосылки и заключения, а также помечено коэффициентом уверенности. Предпосылка обычно представляет собой выражение над фактами. Она может быть выполненной при текущем состоянии рабочей памяти (с соответствующим коэффициентом уверенности). Если она верна, то правило может быть применено. Заключение - это действие, которое можно осуществить, если верна предпосылка. Например, добавить к рабочей памяти некоторый новый факт, коэффициент уверенности которого вычисляется на основе таких же показателей предпосылки и правила. Применение правила состоит в выполнении действия заключения. Совокупность правил называется нечеткой базой знаний.

Прямым выводом в продукционной системе называется процесс применения правил к содержимому рабочей памяти (его исходное состояние задано в начале работы), и соответственно получение в результате новых фактов с их коэффициентами уверенности. Обратныц вывод - это противоположный процесс. В нем по некоторому набору результирующих фактов - гипотезе, путем анализа правил в направлении от заключения к предпосылке, вычисляется коэффициент уверенности гипотезы при заданном исходном содержимом рабочей памяти. Машина вывода - программный модуль, который непосредственно реализует прямой или обратный вывод. 


\section{С. Д. Махортов, М. В. Лещинская}

Предлагаемый подход к исследованию продукционных систем основан на представлении множеств фактов и правил FLP-структурой. Каждый элементарный факт отображается атомом решетки, предпосылка и заключение правила - соответствующими элементами решетки, а правила представляются функцией принадлежности нечеткого бинарного отношения $R$.

Далее для введенного класса алгебраических систем рассматриваются стандартные вопросы теории LP-структур: о логическом замыкании, эквивалентных преобразованиях, канонической форме, логической редукции.

\section{3. ЗАМЫКАНИЕ, ПРЕОБРАЗОВАНИЯ И РЕДУКЦИЯ НЕЧЕТКИХ LР-СТРУКТУР}

Результаты настоящего раздела приведены без доказательств. Они планируются к опубликованию в отдельной статье в математическом журнале.

Заданное на решетке нечеткое бинарное отношение моделирует совокупность нечетких продукций интеллектуальной системы. Оно как правило не является логическим, однако может быть рассмотрено его логическое замыкание. Это замыкание по сути содержит всевозможные логические выводы в продукционной системе.

Логическим замыканием нечеткого бинарного отношения $R$ называется наименьшее логическое отношение, содержащее $R$. Для выяснения вопроса о его существовании введем отношение логической связи пар элементов решетки при заданном $R$.

Определение 3.1. Пусть задано нечеткое отношение $R$ на решетке $\mathbb{F}$. Отношение $\bar{R}$ логической связи пар $A, B \in \mathbb{F}$ определяется функцией принадлежности $\bar{\mu}_{R}$, значение $\bar{\mu}_{R}(A, B)$ которой вычисляется как максимальное из следующих вариантов его вычисления:

1) $\bar{\mu}_{R}(A, B)=\mu_{R}(A, B)$

2) если $A \supseteq B$, то $\bar{\mu}_{R}(A, B)=1$, иначе $=0$;

3) $\bar{\mu}_{R}(A, B)=\min \left(\bar{\mu}_{R}\left(A, B_{1}\right), \bar{\mu}_{R}\left(A, B_{2}\right)\right)$ $\left(\forall B_{1}, B_{2}: B_{1} \cup B_{2}=B\right)$;

4) $\bar{\mu}_{R}(A, B)=\min \left(\bar{\mu}_{R}(A, C), \bar{\mu}_{R}(C, B)\right)$ $(\forall C \in \mathbb{F})$.
Рекурсивное определение 3.1 по данному $R$ задает новое нечеткое отношение $\bar{R}$ на решетке $\mathbb{F}$, которое содержит $R$, 卫, а также обладает некоторыми дополнительными свойствами.

Теорема 3.1. Для произвольного отношения $R$ логическое замыкание существует и совпадает с нечетким отношением логической связи $\bar{R}$, определяемым функцией принадлежности $\mu_{\bar{R}}=\bar{\mu}_{R}$.

Понятие логического замыкания и доказанная теорема о его существовании позволяют рассмотреть вопросы эквивалентности FLP-структур.

Определение 3.2. Два нечетких отношения $R, P$ на общей решетке называются (логически) эквивалентными $(R \sim P)$, если их логические замыкания совпадают. Эквивалентным преобразованием нечеткого отношения $R$ называется такая модификация его функции принадлежности $\left(\mu_{R} \rightarrow \mu_{P}\right)$, что полученное в результате новое отношение $P$ логически эквивалентно $R$.

Определение 3.3. Пусть $R$ - нечеткое отношение на решетке $\mathbb{F}$ с функцией принадлежности $\mu_{R}$ и $M=\left\{\left(A_{t}, B_{t}\right) \mid A_{t}, B_{t} \in \mathbb{F} ; t \in T\right\}$ некоторое множество упорядоченных пар, причем $\min \left(\mu_{R}\left(A_{t}, B_{t}\right)\right)>0$. Пара $(A, B)$ называется (логически) выводимой из $M$, если $\bar{\mu}_{R}(A, B) \geq \min _{t}\left(\mu_{R}\left(A_{t}, B_{t}\right)\right)$.

Следствие 3.1. Пусть $R$ - нечеткое отношение на решетке $\mathbb{F}$ c функцией принадлежности $\mu_{R}, M=\left\{\left(A_{t}, B_{t}\right) \mid A_{t}, B_{t} \in \mathbb{F} ; t \in T\right\}-$ некоторое множество пар, а пара $(A, B)$ выводима из $M$. Определим новое отношение $R^{\prime}$ следующей функцией принадлежности:

$$
\begin{gathered}
\mu_{R^{\prime}}(X, Y)= \\
\qquad \begin{array}{c}
\max \left(\mu_{R}(A, B), \min _{t}\left(\mu_{R}\left(A_{t}, B_{t}\right)\right)\right), \\
\text { при } X=A, Y=B \\
\mu_{R}(X, Y), \quad \text { иначе. }
\end{array}
\end{gathered}
$$

Тогда отношение $R^{\prime}$ эквивалентно $R$.

Справедливо следующее утверждение.

Теорема 3.2. Пусть $R_{1}, R_{2}, R_{3}, R_{4}$ - нечеткие отношения на общей решетке $\mathbb{F}$. Если при этом $R_{1} \sim R_{2}$ и $R_{3} \sim R_{4}$, то $R_{1} \cup R_{3} \sim R_{2} \cup R_{4}$.

$\mathrm{B}$ ее частном случае имеем следующий результат. 
Следствие 3.2. Пусть $R_{1}, R_{2}, R_{3}$ - нечеткие отношения на общей решетке $\mathbb{F}$. Если при этом $R_{1} \sim R_{2}$, то $R_{1} \cup R_{3} \sim R_{2} \cup R_{3}$.

Следствия 3.1-3.2 обосновывают принципы локально-эквивалентных преобразований FLP-структур. Они могут быть, в частности, использованы для эквивалентного упрощения нечетких отношений на решетке.

Нечеткое отношение $R$ на атомно-порожденной решетке $\mathbb{F}$ называется каноническим, если его функция принадлежности положительна лишь на парах вида $(A, a)$, где $A \in \mathbb{F}, a$ - атом в $\mathbb{F}$. Каноническое отношение в моделируемой продукционной системе соответствует множеству правил так называемого хорновского типа.

Теорема 3.3. Для произвольного нечеткого отношения $R$ на атомно-порожденной решетке $\mathbb{F}$ существует эквивалентное ему каноническое отношение.

Рассмотрим еще один важный вопрос, связанный с нечеткими LP-структурами, а именно - их эквивалентную минимизацию.

Логической редукиией нечеткого отношения $R$ на решетке называется любое минимальное отношение, эквивалентное $R$.

Поскольку множество всех нечетких отношений на общей решетке является частично упорядоченным, различаются понятия минимального и наименьшего отношений [11]. В определении логической редукции речь идет о минимальном отношении, поэтому оно может оказаться не единственным.

Следующая теорема указывает достаточное условие существования логической редукции данного отношения. Способ ее построения содержится в доказательстве теоремы, которое будет опубликовано в отдельной статье.

Теорема 3.4. Пусть для нечеткого отношения $R$ на решетке $\mathbb{F}$ построено логическое замыкание $\bar{R}$. Тогда, если для $\bar{R}$ существует транзитивная редукция, то для исходного отношения $R$ существует логическая редукция.

\section{4. РАСПРЕДЕЛЕННАЯ НЕЧЕТКАЯ LP-СТРУКТУРА И ЕЕ СВОЙСТВА}

Предыдущие работы показали [7], что теория LP-структур легко адаптируется для моделирования распределенных логических систем. В этом разделе аналогичный метод применяется для обобщения исследуемых в настоящей статье нечетких LP-структур.

Предположим, что имеется распределенная вычислительная сеть, в каждом узле которой может храниться набор фактов (база данных), подмножество нечетких продукционных правил (база знаний), а также может функционировать машина логического вывода с локальной рабочей памятью. Таким образом, есть распределенная нечеткая продукционная система, состоящая из набора локальных продукционных систем (подсистем), согласованных друг с другом. Под согласованностью понимается выполнение условий, сформулированных в [16].

Каждому факту и каждому правилу распределенной нечеткой продукционной системы сопоставляется подмножество узлов вычислительной сети, на которых они хранятся. Пусть $N$ - множество узлов, $\mathbb{N}$ - порожденный им булеан. Элементарные факты и правила нечеткой продукционной системы будут помечаться элементами решетки $\mathbb{N}$ как атрибутами.

Итак, даны две решетки: $\mathbb{F}$ (образует LP-структуру) и $\mathbb{N}$ (решетка узлов). Ha peшетке $\mathbb{F}$ определено отображение $\operatorname{Nodes}($ ), которое каждому атому $a \in \mathbb{F}$ ставит в соответствие единственный непустой элемент $X \in \mathbb{N}$ (то есть $\operatorname{Nodes}(a)=X, X \neq \varnothing)$ ). Ha peшетке $\mathbb{F}$ задается нечеткое бинарное отношение $R$. Кроме того, каждой паре $A, B \in \mathbb{F}$, для которой $\mu_{R}(A, B)>0$ также сопоставляется непустой элемент $Y \in \mathbb{N} \quad(\operatorname{Nodes}(A, B)=Y$, $Y \neq \varnothing)$.

Для моделируемой продукционной системы функция Nodes() определяет для каждого элементарного факта или каждого правила совокупность узлов распределенной вычислительной системы, где они хранятся.

Определение 4.1. Описанная выше алгебраическая система называется распределенной нечеткой LP-структурой (FDLP-структурой). 


\section{С. Д. Махортов, М. В. Лещинская}

Замечание 4.1. При заданном отображении Nodes() решетка $\mathbb{F}$ и отношение $R$ также могут считаться распределенными.

Для нового понятия FDLP-структуры может быть рассмотрен стандартный круг вопросов, а именно - о замыкании, эквивалентных преобразованиях, канонической форме, логической редукции.

Определение 4.2. Распределенное нечеткое бинарное отношение $R$ на распределенной решетке $\mathbb{F}$ называется продукционно-логическим, если оно рефлексивно, транзитивно и дистрибутивно, причем для каждой пары $A, B \in \mathbb{F}$ справедливо $\mu_{R}(A, B)>0 \Rightarrow \operatorname{Nodes}(A, B)=N$. Логическим замькканием отношения $R$ называется наименьшее продукционно-логическое отношение, содержащее $R$.

Замечание 4.2. Данное определение не означает его применения на практике, то есть совокупность правил распределенной продукционной системы не обязана храниться одновременно на всех узлах. Определение носит теоретический характер и используется для обоснования других результатов, в том числе и с практической точки зрения.

Существование логического замыкания для распределенного нечеткого отношения непосредственно вытекает из соответствующего базового результата (теорема 3.1). Достаточно построить для $R$ логическое замыкание $\bar{R}$ в обычном смысле и пометить каждую пару атрибутом $N$. Таким образом, справедливо следующее утверждение, сформулированное в виде теоремы ввиду его важности, но не сложности доказательства.

Теорема 4.1. Для распределенного нечеткого бинарного отношения $R$ на распределенной решетке существует логическое замыкание $\bar{R}$.

Аналогично и без затруднений на FDLPструктуру переносится концепция эквивалентных преобразований и ее обоснование.

Определение 4.3. Два распределенных нечетких отношения $R, P$ на решетке $\mathbb{F}$ называются эквивалентными $(R \sim P)$, если их логические замыкания (в смысле определения 4.2) совпадают. Эквивалентным преобразованием отношения $R$ называется такая модификация функции принадлежности
$\left(\mu_{R} \rightarrow \mu_{P}\right)$, что новое отношение $P$ эквивалентно $R$.

Из теоремы 3.2 п. 3 и теоремы 4.1 настоящего раздела непосредственно вытекает следующий результат.

Теорема 4.2. Пусть $R_{1}, R_{2}, R_{3}, R_{4}$ - распределенные нечеткие отношения на общей распределенной решетке. Если при этом $R_{1} \sim R_{2}$ и $R_{3} \sim R_{4}$, то $R_{1} \cup R_{3} \sim R_{2} \cup R_{4}$.

Для ее доказательства достаточно к отношениям $R_{1} \cup R_{3}$ и $R_{2} \cup R_{4}$ применить теорему 3.2, после чего в построенных замыканиях двух отношений пометить каждую пару атрибутом $N$.

Замечание 4.3. При выполнении эквивалентных преобразований распределенного нечеткого отношения $R$ значения отображения $\operatorname{Nodes}()$ определяющей роли не играют. Важно лишь, что пары $A, B \in \mathbb{F}$ с положительными значениями старой и новой функций принадлежности имеют непустой атрибут $\operatorname{Nodes}(A, B)$.

Переходим к обсуждению вопросов оптимизации FDLP-структур. С этой целью (аналогично [7]) наряду с функцией Nodes() введем еще два отображения из $\mathbb{F}$ в $\mathbb{N}$. Пусть выбран произвольный элемент $A \in \mathbb{F}$. Поскольку решетка $\mathbb{F}$ является атомно-порожденной, существует представление $A=\bigcup a_{i}$ в виде объединения атомов. Обозначим

$$
\begin{aligned}
& \operatorname{NodesMeet}(A)=\bigcap_{i} \operatorname{Nodes}\left(a_{i}\right) ; \\
& \operatorname{Nodes} \operatorname{Join}(A)=\bigcup_{i} \operatorname{Nodes}\left(a_{i}\right) .
\end{aligned}
$$

В терминологии предметной области можно заметить, что отображение NodesMeet() определяет вычислительные узлы, каждый из которых содержит все элементарные факты, порождающие множество фактов $A$. Отображение NodesJoin() выдает все узлы, хранящие хотя бы один из таких фактов.

Как уже подчеркивалось, точное значение непустого атрибута $\operatorname{Nodes}(A, B)$ несущественно для построения логического замыкания $R$. Но какое оказывается предпочтительным? Ответ дает стратегия распределенного нечеткого логического вывода. Одна из целей моделирования - обоснование снижения трафика между узлами. С другой стороны, неце- 
Алгебрачческая модель распределенной продукиионной системь с нечеткими правилами

лесообразно излишнее дублирование информации на различных узлах.

Пусть на некотором узле работает локальная машина вывода, оперирующая в основном фактами этого узла. Для нее окажется затратным поиск на других узлах сети правил вывода собственных фактов. Априори неизвестно, какие узлы предстоит запросить и в каком порядке. Будем считать целесообразным хранение на узле всех правил, оперирующих фактами этого же узла. Таким образом, аналогично [7] приходим к следующим понятиям «хорошего» распределения правил по узлам вычислительной сети.

Определение 4.4. Распределенное нечеткое отношение $R$ на решетке $\mathbb{F}$ называется релевантно-корректным, если для любых $A, B \in \mathbb{F}$, $\mu_{R}(A, B)>0, \quad$ справедливо $\operatorname{Nodes}(A, B) \supseteq$ $\supseteq \operatorname{NodesJoin}(A) \| N o d e s J o i n(B)$; релевантнонормализованным, если для нее

$$
\begin{gathered}
\operatorname{Nodes}(A, B)= \\
=\operatorname{NodesJoin}(A) \cup \text { NodesJoin }(B) .
\end{gathered}
$$

Определение 4.5. Распределенное нечеткое отношение $R$ на распределенной решетке $\mathbb{F}$ называется каноническим, если оно релевантно-нормализовано, а его функция принадлежности положительна лишь на парах вида $(A, a)$, где $A \in \mathbb{F}, a$ - атом в $\mathbb{F}$.

На основе теоремы 3.3 выводится следующий факт.

Теорема 4.3. Для любого распределенного нечеткого отношения на существует эквивалентное ему каноническое отношение.

Схема ее доказательства аналогична двум предыдущим теоремам.

Далее рассмотрим детали построения логического замыкания отношения. Справедлива теорема, доказываемая по обычной для данного раздела схеме.

Теорема 4.4. Для распределенного нечеткого отношения $R$ логическое замыкание представляет собой отношение $\bar{R}$ (замыкание в смысле п.3), каждая пара которого $A, B$ с $\mu_{R}(A, B)>0$ помечена атрибутом (1).

Наконец, рассмотрим вопрос об эквивалентной минимизации распределенных нечетких бинарных отношений.
Определение 4.6. Логической редукиией распределенного нечеткого отношения $R$ на распределенной решетке называется любое минимальное отношение, эквивалентное $R$ и при этом релевантно-нормализованное.

Следующая теорема указывает достаточное условие существования логической редукции распределенного нечеткого отношения. Способ ее построения содержится в доказательстве теоремы 3.4.

Теорема 4.5. Пусть для распределенного нечеткого отношения $R$ построено отношение логических связей $\bar{R}$ (см. п. 3). Тогда, если для $\bar{R}$ существует транзитивная редукция, то для исходного отношения $R$ существует логическая редукция $R^{0}$. При этом каждая пара $A, B$ отношения $R^{0}$ с $\mu_{R}(A, B)>0$ помечена атрибутом (1).

\section{ЗАКЛЮЧЕНИЕ}

В настоящей работе введен и исследован новый класс алгебраических систем FDLP-структуры, семантика которых охватывает распределенные интеллектуальные системы с нечеткими правилами. Для данного класса рассмотрены стандартные вопросы теории LP-структур: о логическом замыкании, эквивалентных преобразованиях, канонической форме, логической редукции. Представленные теоретические результаты могут быть применены в задачах управления знаниями распределенных нечетких продукционных систем.

Исследование выполнено при финансовой поддержке РФФИ в рамках научного проекта № 19-07-00037.

\section{СПИСОК ЛИТЕРАТУРЫ}

1. Бениаминов, Е. М. Алгебраические методы в теории баз данных и представлении знаний / Е. М. Бениаминов. - М. : Научный мир, 2003. - 184 с.

2. Жожикашвили, А. В. Алгебраическая теория продукционных систем / А. В. Жожикашвили, В. Л. Стефанюк // VIII нац. конф. по искусственному интеллекту с международ- 


\section{С. Д. Махортов, М. В. Лещинская}

ным участием КИИ-2002: Труды конференции. Т. 1. - М. : Физматлит, 2002. - С. 428-436.

3. Maciol, A. An application of rule-based tool in attributive logic for business rules modeling / A. Maciol // Expert Systems with Applications. 2008. - V. 34, \#3. - P. 1825-1836.

4. Дородных, Н. О. Использование диаграмм классов UML для формирования продукционных баз знаний / Н. О. Дородных, А. Ю. Юрин // Программная инженерия. 2015, № 4. - C. 3-9.

5. Махортов, С. Д. Математические основы искусственного интеллекта: теория LP-структур для построения и исследования моделей знаний продукционного типа / С. Д. Махортов ; Под ред. В. А. Васенина. М. : Издательство МЦНМО, 2009. - 304 с.

6. Болотова, С. Ю. Алгоритмы релевантного обратного вывода, основанные на решении продукционно-логических уравнений / С. Ю. Болотова, С. Д. Махортов // Искусственный интеллект и принятие решений. - 2011, № 2. - C. 40-50.

7. Махортов, С. Д. Алгебраическая модель распределенной логической системы продукционного типа / С. Д. Махортов // Программная инженерия. - 2015. - № 12. - С. 32-38.

8. Батыршин, И. 3. Нечеткие гибридные системы: Теория и практика / И. 3. Батыршин, А. О. Недосекин, А. А. Стецко, В. Б. Тарасов, А. В. Язенин, Н. Г. Ярушкина ; Под ред. Н. Г. Ярушкиной. - М. : Физматлит, 2007. $208 \mathrm{c}$.
9. Махортов, С. Д. Оптимизация метода LP-вывода / С. Д. Махортов, А. Н. Шмарин // Нейрокомпьютеры. Разработка, применение. - 2013. - № 9. - С. 59-63.

10. Махортов, С. Д. Нечеткий LP-вывод и его программная реализация / С. Д. Махортов, А. Н. Шмарин // Программная инженерия. -2013 , № 12 . - С. 34-38.

11. Биркгоф, Г. Теория решеток : пер. с англ. / Г. Биркгоф. - М. : Наука, 1984. - 568 с.

12. Рыжов, А. П. Элементы теории нечетких множеств и ее приложений / А.П. Рыжов. - М. : Диалог-МГУ, 2003. - 81 с.

13. Garmendia, L. An Algorithm to Compute the Transitive Closure, a Transitive Approximation and a Transitive Opening of a Fuzzy Proximity / L. Garmendia, R.G. Del Campo, V. López, J. Recasens // Mathware \& Soft Computing, 16 (2009). - P. 175-191.

14. Hashimoto, H. Reduction of a Nilpotent Fuzzy Matrix / H. Hashimoto // Information Sciences, 27 (1982). - P. 233-243.

15. Sowyer, B. Programming Expert Systems in Pascal / B. Sowyer, D. Foster. - John Wiley \& Sons, Inc., 1986. - 186 c.

16. Гинкул, Г. П. Алгоритм цитирования для построения гибридных выводов в продукционных системах / Г. П. Гинкул, С. Ю. Соловьев // Программные системы и инструменты (под ред. Л. Н. Королёва). - МАКС Пресс, 2013. - T. 14. - C. 172-175.

Махортов Сергей Дмитриевич - заведующий кафедрой программирования и информационных технологий, д-р физ.-мат. наук, Воронежский государственный университет, e-mail: msd_exp@outlook.com

Лещинская Мария Владимировна - аспирант кафедры математического обеспечения ЭВМ, Воронежский государственный университет, e-mail: maria-leshchinskaya@mail.ru 


\title{
AN ALGEBRAIC MODEL OF THE DISTRIBUTED PRODUCTION SYSTEM WITH FUZZY RULES
}

\author{
S. D. Makhortov, M. V. Leshchinskaya \\ Voronezh State University
}

\begin{abstract}
Annotation. The algebraic theory of LP-structures is intended for modeling and optimization of production and similar systems in computer science. One of its areas of application is intelligent systems based on rules in production form of. In previous studies, the authors obtained results that substantiate and automate the solution of a number of problems for production systems: equivalent transformations, elimination of redundancy, verification, acceleration of backward inference.

In this paper, we introduce and study the LP structure, the semantics of which incorporates (covers) distributed fuzzy production systems. The terminology of FDLP structures (Fuzzy Distributed LP structures) with a fuzzy binary relation is introduced. A definition of the logical closure of a fuzzy binary relation is given, a theorem on its existence is presented. The theorem allows you to introduce the concept of equivalent FDLP structures, respectively, in applications - equivalent knowledge bases. A theorem on equivalent transformations of an FDLP structure is formulated. Its applied value is the method and its justification for automated transformations of distributed fuzzy knowledge bases. A statement on the reduction of the FDLP structure to a canonical form is also presented. In applications this format corresponds to the set of Horn rules.

Within the framework of the constructed extended model, the possibilities of the theory of LP structures are available in the construction and study of distributed intelligent systems with fuzzy rules.

Keywords: intelligent system, fuzzy productions, LP structure, logical closure, equivalent transformations, logical reduction, distributed system.
\end{abstract}

Makhortov S. D. - Head of the Programming and Information Technologies Department, Doctor of Science, Voronezh State University, e-mail: msd_exp@outlook.com

Leshchinskaya M. V. - postgraduate student of the Applied and System Software Department, Voronezh State University, e-mail: maria-leshchinskaya@mail.ru 